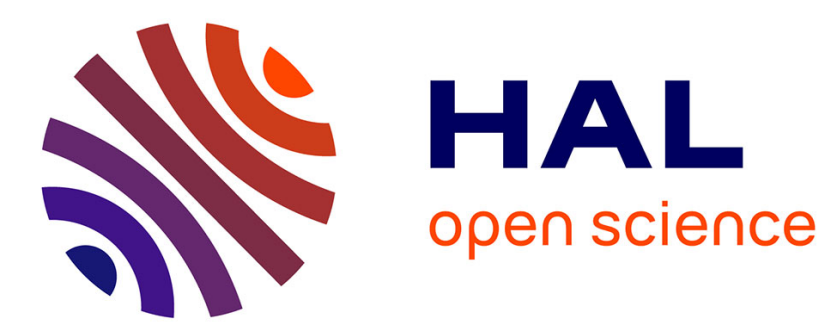

\title{
BORGES, LECTEUR DE KRACAUER
}

Fernando Stefanich

\section{To cite this version:}

Fernando Stefanich. BORGES, LECTEUR DE KRACAUER. Sociétés - Revue des sciences sociales et humaines, 2010, La pensée de Siegfried Kracauer, 110, pp.105-117. 10.3917/soc.110.0105 . hal03558778

\section{HAL Id: hal-03558778 \\ https://hal.science/hal-03558778}

Submitted on 4 Feb 2022

HAL is a multi-disciplinary open access archive for the deposit and dissemination of scientific research documents, whether they are published or not. The documents may come from teaching and research institutions in France or abroad, or from public or private research centers.
L'archive ouverte pluridisciplinaire HAL, est destinée au dépôt et à la diffusion de documents scientifiques de niveau recherche, publiés ou non, émanant des établissements d'enseignement et de recherche français ou étrangers, des laboratoires publics ou privés. 


\section{BORGES, LECTEUR DE KRACAUER}

\section{Fernando STEFANICH}

Jorge Luis Borges et Siegfried Kracauer auraient pu se rencontrer dans l'Europe de la Première Guerre Mondiale. L'un vivait en Allemagne, l'autre en Suisse. Ils partageaient tous deux le goût de la littérature policière, des villes et du cinéma. Borges rentra en Argentine en 1921 et la rencontre n'eut jamais lieu.

Dans les pages qui suivent, nous confronterons la pensée de ces deux intellectuels. D’abord, nous reconstruirons ce dialogue impossible en nous penchant sur la critique de la raison énoncée par Kracauer dans Le roman policier: un traité philosophique, et littérairement par Borges dans des nouvelles telles que « La mort et la boussole », « Le jardin aux sentiers qui bifurquent » ou le recueil Six problèmes pour Don Isidro Parodi. Sachant que Borges avance une critique qui est presque toujours tangentielle, indirecte, nous essayerons de décrypter ses stratégies narratives. Enfin, l’œuvre de Borges et les analyses de Kracauer nous permettront de démontrer que le genre policier représente essentiellement une allégorie de la société contemporaine.

\section{Kracauer et le genre policier}

Siegfried Kracauer fut sans doute le premier intellectuel à comprendre la place qu’occupait la ratio dans les récits policiers et dans la société. Architecte de formation, journaliste de profession, il s’intéressa aux phénomènes de société et se montra aussi à l’aise dans l'analyse du cinéma que du roman policier, à la légitimation duquel il contribua de façon décisive. Sa méthode le conduit du général au particulier : dans Le roman policier, il cherche à saisir la pensée de la société occidentale à partir du genre policier - celui de Conan Doyle et d’Agathe Christie -, considéré comme un tout, sans s’attarder sur les œuvres ni sur les auteurs ; dans De Caligari à Hitler, il cherche à interpréter, au travers des films d'avant-guerre, la société allemande qui permit l'ascension du dictateur. 
Le roman policier ne fut publié qu'après sa mort. L’hypothèse de Rainer Roschlitz est qu'à l'époque où Kracauer achevait l'ouvrage, son ami et collègue Walter Benjamin découvrait le marxisme et que Kracauer, sous son influence, allait suivre le même chemin en oubliant toute sa production antérieure. Roschlitz l'explique ainsi : « C’est en 1925, lorsqu'il achève sa rédaction, que Walter Benjamin rédige son traité sur le drame baroque allemand pour le présenter à l’Université de Francfort ; et c’est cette même année que Benjamin s’oriente vers le marxisme ${ }^{1}$.

L’approche de Kracauer est très particulière, car elle met en rapport le roman policier, la théologie et la philosophie. Cette démarche s’explique par les inquiétudes de l’époque ; elle s'inscrit en effet dans le sillage des travaux de Lukacs et de Benjamin, La Théorie du roman (1916) pour le premier, Les affinités électives de Goethe pour le second, publié en 1922, alors que L’Origine du drame baroque allemand est déjà en préparation. Le déclencheur aurait pu être aussi l'œuvre de G. K. Chesterton dont le principal personnage est un homme d’Église, le père Brown.

Ainsi, rédigé entre 1923 et 1925, Le roman policier est probablement la première analyse sérieuse consacrée au genre. Nous pouvons citer comme antécédents un nombre réduit de titres : De l'assassinat considéré comme l'un de beaux arts (1827), inclassable ouvrage de De Quincey, «Plaidoyer pour les romans policiers » (1901) de Chesterton, « Notes sur Dostoïevski », article que Lukacs rédige en 1914 et qui est une esquisse de la Théorie du roman². «Le Detective novel et l'influence de la pensée scientifique » de Régis Messac, première Thèse universitaire consacrée au genre, ne sera publiée qu'en 1929 et Puissances du roman, où Roger Caillois s'attaque dans l'un des chapitres au roman policier, date de 1941.

Bien plus tard, en 1965, Ernst Bloch publiera Considération philosophique du roman policier. Citons également les travaux d'Ernest Mandel, d'Yves Reuter, de Jacques Dubois et de Todorov, lequel rangea le genre («Typologie du roman policier », Poétique de la prose) dans le rayon des paralittératures. Le cas de Todorov est représentatif d'un secteur de la critique qui n’y voit qu'une littérature d'évasion, tandis que Kracauer parvient à en tirer des observations sociologiques et philosophiques qui mettent en évidence sa complexité. En ce sens, son œuvre s’ajoute à celles de Benjamin, de Gide, de Malraux, de Borges..., auteurs qui ont cherché - et contribué - à légitimer le roman policier.

\footnotetext{
${ }^{1}$ R. Roschlitz, « Avant-propos », Le roman policier : un traité philosophique, p. 9.

${ }^{2}$ Ibid., p. 10.
} 
Dans De Caligari à Hitler, Kracauer explique l’intérêt qui le conduit vers ces modes d'expressions populaires dont le roman policier fait partie : «[...] les revues et les radios populaires, les best-sellers, les modes du langage ainsi que d'autres produits sédimentaires de la vie culturelle d'un peuple donnent également des informations précieuses sur les attitudes prédominantes et autres tendances généralement répandues $»^{3}$.

Kracauer analyse le genre policier en tant que symptôme. S’il s’attaque aux données de surface - « la vie intérieure se manifeste dans divers éléments et conglomérats de la vie extérieure $»^{4}$-, c’est pour comprendre non seulement la société allemande, mais aussi la société contemporaine dans son ensemble - « J'ai certaines raisons de croire que l'utilisation faite ici des films en tant que moyen de recherche pourrait être appliquée avec profit à l'étude du comportement des masses aux États-Unis et ailleurs $»^{5}$ - et pour fonder une sorte d' « histoire psychologique $»^{6}$.

\section{La ratio chez Kracauer}

Construit autour de l'ivresse rationaliste produite par la révolution industrielle, héritage du Siècle des Lumières, le genre à énigme prône la primauté de la ratio sur l'instinct et l'émotion, alors que la réalité historique ne tardera pas à donner des preuves du contraire : une première guerre mondiale, une crise sans précédent (1929), l’arrivée au pouvoir du fascisme... Kracauer anticipe de cette manière les analyses de l'Ecole Critique, celles d'Horkheimer et d'Adorno.

Au début des années vingt, suite au traumatisme de la Première Guerre mondiale et à la révision nietzschéenne, Dieu est donné pour mort - assassiné, pourrions-nous dire, pour rester fidèles au sujet de cet article. Une nouvelle théologie se fait jour, basée sur le culte de la raison, dont le roman policier est le reflet. Ce nouveau culte mis en lumière par Kracauer est organisé autour de la figure du détective. Ce dernier, comme le moine, est un être solitaire, condamné au célibat. Il représente la ratio. Kracauer écrit : «comme Dieu crée l’homme à son image, ainsi la ratio s’engendre elle-même en l’ombre abstraite du détective » ${ }^{7}$.

Le détective de Kracauer ne dépiste pas le criminel parce qu’il aurait agi illégalement ; il ne s’identifie pas non plus aux représentants du principe de légalité. Ce détective dénoue

\footnotetext{
${ }^{3}$ S. Kracauer, De Caligari à Hitler, p. 7.

${ }^{4}$ Ibidem.

${ }^{5}$ Idem, p. VII.

${ }^{6}$ Idem, p. 9.

${ }^{7}$ S. Kracauer, Le Roman policier : un traité philosophique, p. 79.
} 
l'énigme «pour l'amour du processus de déchiffrement »", et s’il se situe du côté de la police, c’est parce que « le légal et l’illégal existent encore en tant que résidus »"

Le fait d’incarner la ratio est précisément ce qui différencie le détective du policier : « À la recherche du criminel, elle [la police] procède rationnellement, en employant tous les moyens légaux, mais d'emblée dans le seul cadre des possibilités qui lui sont données, alors que le détective représente la ratio sans réserve [...] $»^{10}$. Kracauer explique que la police agit motivée par l'intérêt social qui bride l’intellect, tandis que le détective peut s’abandonner au libre jeu de son intelligence.

Cette nouvelle religion fondée sur la ratio s’articulerait ainsi : la messe est célébrée dans le Hall de l'hôtel, le mystère à élucider correspond au Mystère divin, le processus de résolution de l'énigme est une œuvre de communion grâce à laquelle le prêtre-détective aide le pêcheur à rallier la loi.

Une autre caractéristique de ces récits est qu’ils se terminent toujours par « la victoire incontestée de la ratio ${ }^{11}$. «Pas de roman policier - nous dit Kracauer - dont le détective ne finisse par éclairer l'obscurité et par reconstituer sans lacune la banalité des faits ${ }^{12}$. Ainsi, l’ordre momentanément perturbé est finalement rétabli.

\section{Kracauer et la transition}

On aurait tort de lire l'ouvrage de Kracauer comme un éloge de la raison, celle-ci ayant sous sa plume - Roschlitz l'a bien compris - une forte connotation négative. Non sans ironie, Kracauer se livre en effet à un dialogue kantien. Il relève le décalage qui existe entre le récit à énigme (héritier des Lumières) et la société contemporaine, le genre policier étant construit sur une structure qui ne correspond plus à la réalité. Cette thèse audacieuse au centre de $L e$ roman policier expliquerait en partie que ce travail soit resté inédit. Pourtant, les faits historiques donneront raison à Kracauer: les temps modernes étant révolus, le postmodernisme s'impose. Ces transformations sociales finiront par entraîner la transformation du roman policier lui-même, le roman noir (postmoderne) se substituant au roman à énigme (moderne).

\footnotetext{
${ }^{8}$ Ibid., p. 121.

${ }^{9}$ Ibid., p. 21.

${ }^{10}$ Ibid., p. 96.

${ }^{11}$ Ibid., p. 173.

${ }^{12}$ Ibid., p. 173.
} 


\section{Le détective chez Borges : Parodi et Lönnrot}

La Première Guerre mondiale achevée, Borges rentre dans son pays. Le milieu culturel de l’Argentine des années vingt est en pleine mutation ; la parole s’est démultipliée et le marché offre un grand nombre de publications périodiques. Dès son retour, Borges commence à collaborer dans diverses revues où il publie ses critiques cinématographiques, littéraires, puis ses premiers textes. Il réussit à concilier les opposés, d'une part en participant à la populaire Revista Multicolor de los Sábados (appartenant au journal Crítica) ainsi qu’à la prestigieuse revue Sur (crée en 1931 par Silvina Ocampo), d’autre part en s’intéressant à la paralittérature sans abandonner pour autant ses préoccupations métaphysiques.

Le cinéma est l'un des passe-temps du jeune Borges. Il a une prédilection pour les westerns et les films de gangsters, en particulier ceux de Von Sternberg, dont il considère qu’ils ont remplacé la poésie épique. Sa passion pour le cinéma le conduira même à écrire des scénarii.

Sa prose, d’abord timidement exposée dans les pages des revues, se consolide dans les années quarante, époque où il publie Fictions et, en collaboration avec son ami Bioy Casares, Six problèmes pour don Isidro Parodi. Les hybridations littéraires de Borges combinent l'intrigue policière, la métaphysique et la littérature gauchesque. Grâce à ces produits kitsch (où le Haut côtoie le Bas), il devient un précurseur du post-modernisme littéraire, sensibilité qui se manifeste également à travers son désenchantement, exprimé (comme toujours chez Borges) non de manière directe, mais tangentielle : derrière les réflexions sur le temps, sur l'unicité et la multiplicité, les textes de Borges constatent l’inutilité de la raison dans le monde contemporain.

De la production policière de Borges naissent deux détectives : Isidro Parodi et Eric Lönnrot. Le premier résout les énigmes sans quitter sa cellule. Enfermé pour un crime dont il est innocent - la police ayant laissé courir le vrai coupable, protégé par les politiciens en place -, Parodi n’a pas besoin d'interroger les suspects ; il n’a pas besoin non plus de voir la scène du crime ; il incarne la ratio menée à son paroxysme. Le second est présenté dans « La mort et la boussole » comme un enquêteur célèbre, au grand pouvoir de déduction. Il enquête sur l'assassinat d'un spécialiste de la kabbale, survenu dans un hôtel alors que le Tétrarque de Galilée, en possession de coûteux saphirs, séjourne dans la chambre contiguë. À une distance symétriquement régulière, dans l'espace et dans le temps, deux autres assassinats sont commis. Grâce à sa ratio, Lönnrot résout l'énigme, mais cette résolution le conduira à sa propre mort. 
Ces deux enquêteurs, malgré leur génie, paraissent être punis. L’un est emprisonné pour un crime qu'il n’a pas commis ; l'autre est assassiné. Leur cas n’est pas isolé. Stephen Albert par exemple, dans «Le jardin aux sentiers qui bifurquent», a consacré sa vie à conserver et à déchiffrer le labyrinthe de Ts’ui Pên. Au dénouement, Albert est assassiné pour que l'espion puisse accomplir sa mission et transmettre enfin son message.

\section{Borges face à la réalité : « La fiesta del monstruo »}

Kracauer avait ouvert la voie. En 1929, Régis Messac publie sa Thèse « Le Detective Novel et l'influence de la pensée scientifique ». Un peu plus tard, un économiste, Ernest Mandel, assure la relève. Dans Meurtres exquis, il se propose d'analyser le genre policier sous l'optique du matérialisme historique. Si Kracauer voit dans la figure du détective la personnification de la ratio, Mandel, lui, l'associe à la mentalité bourgeoise et à l’idée d'ordre. Au-delà des controverses à propos de sa paternité, Mandel place à l'origine du genre policier les récits centrés sur le bon bandit, personnage qui incarnait la lutte du bourgeois contre l'aristocratie. Si le bon bandit est sympathique, c’est parce que sa lutte est légitime. Plus tard cependant - la bourgeoisie ayant pris le pouvoir - l'image du criminel change : il devient celui qui attente à la propriété privée, et dès lors le bourgeois ressent la nécessité de se protéger et de défendre ses intérêts contre lui. Les analyses de Mandel nous placent donc au centre de la lutte de classes.

On sait que les critiques de Borges étaient toujours tangentielles, obliques, et l'on connaît aussi sa volonté de maintenir la politique hors de la littérature. Toutefois, l'une et l'autre vont se mêler de façon assez explicite, directe, voire violente, dans « Le simulacre » et dans « La fiesta del monstruo ». Cette dernière nouvelle, écrite en collaboration avec Bioy Casares sous le pseudonyme de Bustos Domecq, fut publiée le 30 septembre 1955 dans le journal Marcha de Montevideo (symboliquement, cela est important, Montevideo ayant servi de refuge aux opposants du dictateur Juan Manuel de Rosas). La nouvelle décrit un jour férié, jour de fête, où un groupe de partisans de Perón avance depuis le Sud de Buenos Aires pour écouter la parole du leader. En route, la horde péroniste va assassiner un intellectuel juif. Dans cette réécriture d’El matadero (1839) d’Esteban Echeverría, Perón - le « monstre » désigné par le titre - prend la place du «tyran » Juan Manuel de Rosas et l'intellectuel juif celle du « civilisé » unitaire. Dotée d'une forte charge symbolique, la nouvelle est aussi très visuelle. Les masses qui participent à la festivité dionysiaque proviennent des quartiers pauvres et barbares et se dirigent vers le centre pour se l'approprier. Un mouvement similaire se produit 
dans « Maison occupée » de Julio Cortázar ${ }^{13}$. Borges et Bioy Casares expriment, dans cette description de l’Argentine sous domination péroniste, leur rejet du populisme autoritaire.

\section{Matérialisme historique : Apollon et Dionysos}

À la lumière de cette nouvelle, il n’apparaît pas déraisonnable d'étudier l'œuvre de Borges sous une optique sociologique. Pour y parvenir, il nous faut rappeler quelques éléments d’Histoire. Dès ses origines, le pays fut déchiré entre Unitaires et Fédéralistes, qui s’opposaient à la fois par leur vision culturelle de l'Argentine ainsi que par leur conception de son organisation politique. Les Unitaires luttaient pour un pays centralisé, dont la capitale serait Buenos Aires. Leurs modèles étaient les pays européens. Les Fédéralistes possédaient une vision localiste, tant de la vie politique que de la vie culturelle. L’Unitaire Sarmiento synthétisa l'expression de ce clivage dans la formule désormais emblématique « civilisation et barbarie » par laquelle il désignait deux forces, l’une ordonnatrice qui cherchait à organiser le pays, l'autre chaotique et séditieuse. Cette tension entre ces formes apolliniennes et dionysiaques marquera toute l'Histoire nationale.

À partir de 1880, l’Argentine fut gouvernée par une élite, une oligarchie moderniste et pro-européenne. Cette oligarchie est communément appelée Generación del 80. Méprisant le localisme, la Generación del 80 se proposa de faire de Buenos Aires une ville européenne : de larges avenues furent tracées, des monuments, des palais édifiés. Lors de sa visite dans la capitale argentine, Georges Clémenceau déclara que Buenos Aires était une ville européenne $^{14}$. L’imaginaire social - tel que Castoriadis le définit - de la Generación del 80 avait modelé non seulement la ville, mais encore l’habitant. En 1910, le pays fête le Centenaire de son indépendance, et en 1912 est édictée la loi Saenz Peña qui cherche à consolider la démocratie en instaurant le vote universel et secret.

En contrepartie, l’immigration étrangère (promue et encouragée par le gouvernement de la Generación del 80), gagnée aux idées socialistes et anarchistes, entame des luttes pour améliorer les salaires et les conditions de travail. Grèves, manifestations, confrontations se succèdent. Citons à titre d'exemple la «Semaine Tragique » (1919) et la « Patagonie Tragique » (1921), mouvements populaires qui eurent lieu sous la présidence d’Hipólito Yrigoyen et dont la répression violente provoqua des milliers de morts. À cette époque, l'illusion d'une Argentine européenne commence à s'évanouir; le désenchantement

\footnotetext{
${ }^{13}$ La nouvelle, qui avait été publiée par Borges, accepte plusieurs lectures. Elle est née d'un cauchemar et Cortázar se penche pour l'inceste des personnages (ils sont frère et soeur), mais il n'écarte aucune possibilité.

${ }^{14}$ G. Clémenceau, Notas de viaje por la América del Sur: Argentina, Uruguay, Brasil, Buenos Aires, Hispamérica, 1996, p. 26.
} 
s’accentue encore lors de la «Década Infame » pendant laquelle l'instabilité politique le dispute à la fraude et à la violence. Les idées populaires et nationalistes gagnent du terrain, la classe ouvrière s'organise et se consolide ; ce processus aboutit en 1946 à l'élection de Juan Domingo Perón à la présidence.

Le pouvoir change alors de mains. Borges, noble déclassé, sans fortune, qui revendique des origines aristocratiques - dans Essai d'autobiographie, il nous délivre tout un panthéon composé par une ascendance de héros mineurs de l'indépendance argentine -, oppose à cette réalité cauchemardesque (« J’écris en juillet de 1940 ; chaque matin la réalité ressemble davantage à un cauchemar ${ }^{15}{ }^{15}$ ) une stratégie d'évasion et de récupération en développant une littérature anachronique. Borges valorise ainsi l’ancien contre le nouveau : l'ancien qui s'incarne dans le gaucho et la campagne paisible, le couteau, le culte du courage et l'amitié, tandis que le nouveau se manifeste dans les immigrés et les travailleurs urbains, organisés et syndicalisés, qui aux yeux de Borges dégradent le présent en chaos et en désordre.

\section{Évasion : ville et anachronisme}

Chesterton, qui voyait dans le genre policier l'Iliade de la grande ville, admirait la façon dont Conan Doyle faisait ressortir le caractère humain des pierres et des tuiles ${ }^{16}$. En effet, les récits policiers, littérature de la ville et littérature de la crise, sont des outils précieux pour étudier les phénomènes urbains. Siegfried Kracauer décrivait ainsi l’Alexanderplatz : « [...] trois gares souterraines s’y superposent, reliées par un labyrinthe de couloirs, de paliers et d’escaliers $[\ldots]^{17}$ ». Mise au jour des bifurcations, de la multiplicité, de la complexité citadine, la description se poursuit :

À proximité immédiate, visible depuis la place, se dresse un immeuble de bureaux [...]. Les étroites bandes qui les séparent sont occupées par des fenêtres, qui forment comme des crevasses ; ce sont des fenêtres mal nourries, intimidées, qui font penser à des employés. Leur activité est complètement rationalisée, leur salaire parfaitement conforme à la grille de rémunération. Bientôt on les démolira, l'entreprise continuera sans eux et les liserés barreront plus brutalement encore la façade aveugle ${ }^{18}$.

\footnotetext{
${ }^{15}$ J. L. Borges, Sur, n 70 , julio 1940, p. 62.

${ }^{16} \mathrm{M}$. Lits, Le roman policier : introduction à la théorie et à l'histoire d'un genre littéraire, p. 83.

${ }^{17}$ S. Kracauer, « Der neue Alexanderplatz », Schriften, 1971, t. 5-3, p. 150.

${ }^{18}$ Ibid., p. 12.
} 
Kracauer révèle la relation entre la pensée et l'architecture, la ville comme érection de la pensée, la verticalité comme victoire sur la nature, par conséquent comme domination. Nous trouvons chez Nietzsche un antécédent: «Si nous voulions, si nous osions construire une architecture conforme à la nature de notre âme (nous sommes trop lâches pour cela !) le labyrinthe devrait être notre modèle ${ }^{19}$.

Au début du siècle, Buenos Aires est déjà une mégalopole, mais Borges décide de se placer, et de placer son œuvre, ailleurs. Il s'éloigne de l'irratio de la société postmoderne et produit une littérature volontairement anachronique qui a pour protagoniste, non l'homme postmoderne, aliéné et fragmenté, mais le gaucho, et pour cadre, non la mégalopole, mais les faubourgs calmes et vides: «J'imagine Buenos Aires d’une façon anachronique; sans le vouloir j’imagine Buenos Aires comme une ville aux maisons basses [...]. À présent je sais que cette vision est fausse. Et pourtant je continue à la voir [...] $»^{20}$.

Borges connaît la réalité, mais il décide de l'ignorer : «[...] avant la barbarie correspondait à la campagne, et la civilisation, comme l'étymologie nous l'indique, à la ville. Aujourd'hui, il semble que nous ayons aussi une barbarie de la ville, fruit de l'industrialisation $»^{21}$.

À son retour en Argentine, Borges chante sa ville, aussi bien dans ses recueils poétiques que dans ses essais. Pourtant, il ne voit pas le nouveau Buenos Aires, le village barbare devenu une grande cité dont la langue même, le lunfardo ${ }^{22}$, trahit l’influence récente de l'immigration. Rien de cela n'apparaît en effet dans ses textes. Au contraire, l'auteur nous transporte dans les faubourgs, à la frontière qui sépare la ville industrialisée de ce grand vide qu'est la Pampa. Ernst Bloch nous apprenait que «des temps plus anciens que ceux d'aujourd'hui continuent à vivre dans des couches plus anciennes ${ }^{23}$. En effet, «tous ne sont pas présents dans le même temps présent. Ils n’y sont qu'extérieurement, parce qu’on peut les voir aujourd'hui. Mais ce n'est pas pour cela qu'ils vivent en même temps que les autres $»^{24}$. À ce titre, l'amour des ruines et la mort comme ressort dramatique manifestent l'existence de résonances entre la production de Borges et celle du décadentisme ${ }^{25}$.

La cartographie proposée par Borges correspond moins à la réalité qu'à l'imaginaire. C'est un territoire vide et immobile, c'est le territoire du gaucho. Le regard nostalgique de

\footnotetext{
${ }^{19}$ F. Nietzsche, Aurore, Euvres, t.1, p. 169.

${ }^{20}$ J.L. Borges, Borges en dialogue, p. 87.

${ }^{21}$ Ibid., p. 169.

${ }^{22}$ Argot popularisé par le tango.

${ }^{23}$ E. Bloch, Héritage de ce temps, p. 95.

${ }^{24}$ Ibid., p. 95.

${ }^{25}$ J. J. Sebreli, « Borges : nihilismo y literatura », p. 98.
} 
Borges ignore la capitale contemporaine et fait renaître la cité qui précède le modèle agroexportateur et commercial de la Generación del 80.

\section{Le récit policier comme allégorie de la société contemporaine}

\section{La réalité et le labyrinthe}

L’œuvre borgésienne accepte une multiplicité de lectures. Dans Leçons américaines, Italo Calvino en a fait l'éloge en notant que « Le jardin aux sentiers qui bifurquent », qu'il qualifie de vertigineux essai sur le temps, se présente comme un récit d'espionnage, qui à son tour inclut la description d'un immense roman chinois, le tout concentré en une douzaine de pages $^{26}$. C'est dire à quel point l'écriture borgésienne parvient à se ramifier, à se multiplier, tout en préservant une étonnante économie de moyen. Cette capacité à associer complexité et sobriété pourrait trouver un écho dans la figure du labyrinthe, au sujet de laquelle la critique borgésienne a déjà beaucoup écrit.

Il n’y a pas un labyrinthe chez Borges, mais des labyrinthes : le labyrinthe comme ordre, comme espérance, comme organisation dotée d'un centre, mais également le dédale dont on ne peut pas s'échapper, comme chaos, comme ramification, comme bifurcation indéfinie, comme temps... Tel est le cas par exemple du labyrinthe textuel des Mille et une nuits. Dans un entretien, Borges confiait à Osvaldo Ferrari que son cauchemar le plus fréquent était le cauchemar du labyrinthe : « je songe que je suis dans un lieu quelconque et, pour un motif inconnu, je veux sortir de ce lieu. Je parviens à m’échapper, mais je me retrouve dans un lieu exactement semblable, ou le même lieu $»^{27}$.

Cet archétype spatial représente ainsi une clé privilégiée pour interpréter ses textes. Dans le contexte des années trente et quarante, il illustre l'ordre bourgeois et sa ratio, menacés par les forces de l'irratio, par l'avance du prolétariat, des hordes de Perón. L’image d'un Isidro Parodi rationnel, enfermé dans sa cellule, est en ce sens assez explicite. Mais l’image du dédale est aussi une allégorie de la complexité de la société contemporaine où l’homme est prisonnier des faux-semblants, des voiles qui cachent la réalité.

\section{Le simulacre : frontière entre réalité et fiction}

Dans « Le simulacre », un étranger arrive dans un village, met une poupée dans une boîte en bois et organise ses funérailles. Il adopte une attitude solennelle face à la foule qui vient saluer

\footnotetext{
${ }^{26}$ I. Calvino, Leçons américaines, p. 187-188.

${ }^{27}$ J.L. Borges et O. Ferrari, Borges en dialogue, p. 112.
} 
la défunte. Ainsi, Borges offre une parodie des funérailles d'Evita. Après la chute de Perón, dans le numéro 237 de la revue Sur, l'auteur publia un article intitulé « L’illusion comique » où il proposait sa lecture - ainsi que celle du secteur de la société dont il était le porte-parole du phénomène péroniste, décrit comme un cauchemar, un carnaval, une fiction théâtrale construite autour de deux histoires : la première, le délit ; la deuxième, la fiction. C'est cette dernière qui nous intéressera ici. Comparé en effet à une illusion comique, le péronisme produit une fiction faite de «fables pour les dupes $»^{28}$. Notons que les métaphores utilisées pour décrire le phénomène appartiennent toutes au monde du spectacle. Rien d'étonnant à cela si nous nous rappelons qu'à l'époque, le fascisme - dont le péronisme puise une certaine inspiration esthétique - a transformé la politique en spectacle de masse.

Borges interroge souvent la notion de frontière, frontière dans la ville, entre haute culture et culture de masse, mais surtout entre réalité et fiction. D’ailleurs, dans son œuvre, réalité et fiction se confondent sans cesse. La réalité est narration, récit, pur discours. « El Sur » et «Les ruines circulaires » en sont de bons exemples. Dans « De la rigueur de la science », nouvelle publiée dans Historia universal de la Infamia, Borges décrit un Empire où la cartographie était si développée que la carte d'une région occupait une ville, et celle d'un pays, une région. La carte est si détaillée qu’elle finit par recouvrir très exactement le territoire représenté ; le double finit par se confondre avec le réel. Jean Baudrillard voit dans la nouvelle de Borges une « allégorie de la simulation ${ }^{29}$ et l'utilise comme point de départ pour sa réflexion sur la société contemporaine (il en fera autant dans Carnaval et cannibale et Le crime parfait avec une autre nouvelle de Borges : «La Faune des miroirs » ${ }^{30}$ ). Pour le philosophe français, les simulacres se sont substitués à la réalité. Walter Benjamin avait déjà abordé le sujet en parlant de la copie de l'œuvre d'art (à l'époque de sa reproductibilité technique). Mais, tandis que la copie nous renvoie à un original, les simulateurs tentent de faire coïncider le réel avec leurs modèles de $\operatorname{simulation}^{31}$. Nous sommes loin de la copie et de la parodie ; pour que ces simulacres soient tenus pour la réalité, ils nécessitent l'abolition du spectaculaire - pour cela il suffit de suivre l'évolution du péronisme - et par conséquent d'une socialité hyperréaliste. Lorsque le réel n’est plus ce qu’il était, la nostalgie prend tout son sens. On assiste alors à une surenchère des mythes d'origine et des signes de réalité, à une

\footnotetext{
${ }^{28}$ J. L. Borges, «L'illusion comique », Sur, n ${ }^{237}$, nov/déc. 1955, p. 9-10.

${ }^{29}$ J. Baudrillard, Simulation et simulacres, p. 9.

${ }^{30}$ Baudrillard fait appel à un autre texte de Borges pour fonder ses théories, «Le peuple des miroirs » : Les vaincus, relégués de l'autre côté des miroirs, sont réduits à la ressemblance, à n'être plus que l'image-reflet de leur vainqueur... Mais, dit Borges, voilà que peu à peu ils se mettent à leur ressembler de moins en moins et, un jour, ils refranchiront le miroir dans l'autre sens et mettront fin à l'hégémonie de l'Empire [...] ", Carnaval et cannibale, p. 14.

${ }^{31}$ Ibid., p. 10.
} 
escalade du vrai, du vécu ${ }^{32}$. Ce recours nostalgique aux mythes d’origine est ainsi une réponse dialectique, et logique, à l'effondrement du réel. C'est pour cela que Borges fait table rase du présent pour revenir au passé et aux mythes fondateurs : le gaucho et la terre.

Domaine des faux-semblants, le simulacre est l'un des éléments centraux du genre policier. Le lecteur de récits policiers sait que les apparences sont trompeuses et que rien de ce qu’on vit n’est réel. Dans la nouvelle intitulée « Emma Zunz », Emma décide d’assassiner son patron, Loewenthal, afin de venger la mort de son père. Ayant minutieusement planifié sa vengeance, elle couche avec un marin et rend visite à Loewenthal sous prétexte de dénoncer des employés qui comptent lancer une grève. Lorsque la police arrive sur la scène du crime, Emma instaure une nouvelle réalité : en déguisant l'assassinat en légitime défense, elle déclare avoir été violée par Loewenthal. C’est la version que la police retiendra. « La mort et la boussole » est également construite autour de l’idée du simulacre, la série d’assassinats n’étant qu’une mise en scène grâce à laquelle Scharlach exécute sa vengeance.

\section{L'ère du soupçon}

Domaine des faux-semblants, le soupçon est l'un des éléments centraux du genre policier. L’ère de l’innocence étant révolue, le genre policier instaure en effet la suspicion, la méfiance. Dans une conférence dictée à l’Université de Belgrano ${ }^{33}$, Borges imagine la réaction d'un amateur de récits policiers à la lecture de la première ligne du Quichotte : «Dans un village de la Manche, dont je ne veux pas me rappeler le nom [...] ». Ce lecteur de nature suspicieuse ne croira pas d'abord que l'action se soit réellement déroulée dans la Manche ; puis, il se demandera pourquoi Cervantès tait le nom de ce lieu. Cache-t-il sa culpabilité, un meurtre ?

Simulacres, méfiance, énigme, le récit policier est une métaphore de la société contemporaine. La société bourgeoise du capitalisme tardif, la société des monopoles, fonctionne alors comme un grand mystère.

\section{L'énigme}

La réalité comme un grand mystère, telle est également l'idée d’Ernst Bloch : « vous êtes là à travailler assidûment à votre petite affaire, et tout d'un coup cette affaire s’écroule, pour de mystérieuses raisons, sans que vous y soyez pour quelque chose $»^{34}$. Telle est aussi la pensée de Bertolt Brecht :

\footnotetext{
${ }^{32}$ J. Baudrillard, Simulacres, op. cit., p. 17.

${ }^{33}$ J. L. Borges, « El cuento policial », Borges oral, p. 83-105.

${ }^{34}$ E. Mandel, Meurtres exquis, p. 95.
} 
Nos expériences, dans la vie, se font sous forme de catastrophes. C'est de catastrophes que nous devons déduire la manière dont fonctionne notre système social. C'est à l'occasion de crises, de dépressions économiques, de révolutions et de guerres que nous devons, par la réflexion, discerner l'inside story. Déjà en lisant les journaux (mais aussi les notes à payer, les lettres de licenciement, les ordres d'appel sous les drapeaux, etc.), nous sentons que quelqu'un a dû faire quelque chose pour que la catastrophe visible ait eu lieu. Alors, qui a fait quoi ? Derrière les événements qui nous sont annoncés, nous en supposons d'autres qui ne le sont pas. Ceux-ci sont les vrais événements. C’est seulement si nous les connaissions que nous comprendrions. Seule l'histoire peut nous renseigner sur ces vrais événements ; dans la mesure où les acteurs n'ont pas réussi à les tenir tout à fait secrets. L'histoire s'écrit après les catastrophes [...]. L'existence dépend de facteurs inconnus ${ }^{35}$.

Lorsque la réalité est pur discours, lorsque le pouvoir appartient aux grands monopoles et la domination se cache derrière le consensus, l'homme ordinaire perd les repères et doit, comme un détective, dé-voiler, si possible, la réalité, enlever les voiles de l'apparent jusqu’à retrouver le réel (la vérité comme aleteia). Le doute du lecteur du genre policier est finalement le doute de l'homme contemporain.

\section{Conclusion}

Entre 1923 et 1925, Siegfried Kracauer s’attaquait à un sujet mineur, le roman policier. Jusqu’alors, personne ne s’y était intéressé. Quelques décennies plus tard, même s’il existe encore des critiques qui continuent de le ranger dans le rayon des paralittératures, il est entré dans les universités. Kracauer fut ainsi le premier à mesurer le potentiel du genre. Il ouvrit la voie. Si nous n’avons considéré ici que quelques lignes générales, nous pouvons en effet admettre aujourd'hui que le genre policier offre un matériel particulièrement riche pour analyser, par exemple, la ville et la lutte des groupes sociaux pour les espaces et les ressources ${ }^{36}$.

Le roman policier dénonce le décalage existant entre la production littéraire et la société de l'entre-deux-guerres. Alors que Kracauer renouvelle ainsi la compréhension des faits sociaux et littéraires, à des milliers de kilomètres, Jorge Luis Borges s'apprête à entreprendre une tâche similaire. Poète avant-gardiste à ses débuts, il devient ensuite critique, auteur, essayiste, scénariste, éditeur. Motivé par l'avancée de l'irratio propre aux forces

\footnotetext{
${ }^{35}$ E. Mandel, Meurtres exquis, p. 95.

${ }^{36}$ Pour cela je renvoie le lecteur à mon article «La literatura policial argentina en tiempos de crisis : década infame y posmenemismo », Escrituras policíacas : la Historia, la memoria, vol. 2, dir. Dante Barrientos Tecún, Univ. de Provence, 2009, p. 257-271.
} 
dionysiaques du péronisme, il développe une littérature de l’anachronisme, traversée par la polysémie et la critique tangentielle. Le philosophe allemand et l'écrivain argentin ont des intérêts communs : la ville, le cinéma, le genre policier. Pourtant, s’ils coïncident dans le diagnostic, leurs idées sont opposées. Le premier découvre le marxisme, tandis que le second, au contraire, s’affilie au parti conservateur, une façon de nihilisme, dira-t-il. Cette opposition au régime de Perón lui fera saluer plus tard les dictatures, de Lonardi d'abord, de Videla ensuite.

Dans le labyrinthe du temps, les chemins convergent et bifurquent. Dans l'un d'eux, le nôtre, ils ne se sont jamais rencontrés. En analysant l'œuvre de Borges à la lumière de celle de Kracauer nous avons feint cette rencontre, une rencontre impossible entre ces deux intellectuels si proches et si lointains à la fois.

Nous vivons aujourd’hui l'ère du récit et, par conséquent, l’ère du soupçon. Si la figure littéraire du détective a quasiment disparu, c'est parce que désormais le détective est l’homme ordinaire ; le détective, c'est nous. La réalité est devenue en fin de compte un simulacre, une énigme à déchiffrer.

\section{Bibliographie}

Baudrillard, J., Simulation et simulacres, Paris, Galilée, 1981.

Bloch, E., L’héritage de ce temps, Paris, Payot, 1977.

Borges, J. L., Euvres complètes, Paris, Gallimard, 1993.

Borges, J. L., Nuevos cuentos de Bustos Domecq, Buenos Aires, ediciones Librería de la Ciudad, 1997, pp. 87-103.

Borges, J. L., « El cuento policial », Borges oral, Buenos Aires, Emecé, 1989.

Borges, J. L., « L’illusion comique », Sur, n² 237, novembre/décembre 1955, pp. 9-10.

Borges, J. L. et O. Ferrari, Borges en dialogue, Paris, Ed. de l’Aube, 1992.

Calvino, I., Leçons américaines, Paris, Seuil, 2001.

Clémenceau, G., Notas de viaje por la América del Sur : Argentina, Uruguay, Brasil, Buenos Aires, Hispamérica, 1996,

Kracauer, S., Le roman policier : un traité philosophique, Paris, Payot, 1981.

Kracauer, S., De Caligari à Hitler, Lausanne, Ed. l’Age d’homme, 1973.

Kracauer, S., « Der neue Alexanderplatz », Schriften, Frankfurt/M., Suhrkamp, 1971. 
Lits, M., Le roman policier : introduction à la théorie et à l'histoire d'un genre littéraire, Liège, Éditions du CÉFAL, 1993.

Mandel, E., Meurtres exquis, Paris, La Brèche, 1986.

Nietzsche, F., Oeuvres, t. 1, Pairs, R. Laffont, 1993.

Sebreli, J. J., « Borges : nihilismo y literatura », Cuadernos hispanoamericanos, n 565-566, juillet-août 1997. 